\title{
Accelerated Orthodontic Tooth Movement: A Review
}

\author{
Gurbax Singh" ${ }^{1 *}$, Raahat Vikram Singh ${ }^{1}$, Roopsirat Kaur ${ }^{2}$ and Devinder Preet Singh ${ }^{2}$ \\ ${ }^{1}$ Department of Orthodontics and Dentofacial Orthopedics, Maharishi Markandeshwar College of Dental Sciences and Research, India \\ ${ }^{2}$ Department of Orthodontics and Dentofacial Orthopedics, Dr. Harvansh Singh Judge Institute of Dental Sciences and Hospital, India
}

*Corresponding author: Gurbax Singh, BDS MDS (Consultant), L8/34 Gurdev Nagar, TaranTaran Road, Amritsar, India

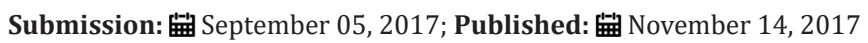

\begin{abstract}
In today's quick paced time and age, the duration of orthodontic treatment has become a primary concern for most patients seeking the treatment. As clinicians too, concerns arose regarding the long term disadvantages of conventional orthodontic treatment like predisposing the patient to caries, gingival recession and resorption of roots. Therefore, the need for a faster treatment with least possible disadvantages arose. Orthodontic treatment involves the response of the tissues surrounding the tooth on which the force is being applied that happens on a chemical, cellular and mechanical level. So to enhance the body's response to these orthodontic forces, various ways were found to accelerate the treatment. This article reviews the biology of tooth movement and discusses various methods like surgical methods-osteotomy and corticotomy and physical methods like low level lasers, direct electric current effect and their promising results along with their limitations.
\end{abstract}

Keywords: Accelerated tooth movement; Corticotomy; Osteotomy; Low level laser therapy; Piezocision; Direct electric current

\section{Introduction}

Today, a plethora of the population undergoes orthodontics treatment in order to bring about better occlusion, improved oral function as well as harmonized facial appearance. However, a perplexing challenge that has not been completely solved in clinical orthodontics is the prolonged treatment time (on an average 2-3 years). Overcoming this challenge will not only dramatically improve the quality of orthodontic care, but also motivate more people towards the concept of orthodontic treatment.

Due to an increased initiative taken by adults, researchers now focus on finding methods to accelerate tooth movements thereby providing a shorter treatment time. Owing to the fact that the metabolism in adults is much slower than those in younger patients the time taken for treatment in adults is significantly greater than that taken in adolescents. The most obvious and possibly the best solution to shorten treatment time are to speed up the rate of tooth movement. Other than the fact that prolonged treatment time causes inconvenience to the patient it also poses several disadvantages like a higher predisposition to caries, gingival recession and root resorption [1].

Numerous attempts have been made to achieve a higher rate of tooth movement, both in vivo as well as in vitro. There are however, many uncertainties and unanswered questions that persist in regard to this field till this day.

Methods to accelerate orthodontic tooth movement can be broadly studied under the following categories [2]:
1. Biological Approach.
2. Surgical Approach.
3. Physical Approach.

\section{Biological approach}

Extensive experiments have been carried out exogenously on molecules such as prostaglandin E (PGE2), cytokines that include lymphocytes and monocytes-derived factors, receptor activator of nuclear factor kappa B ligand (RANKL), and macrophage colonystimulating factor (MCSF) in order to create ways to enhance tooth movement both in animal experiments and humans [3-5].

Effect of cytokines on tooth movement: High concentration of cytokines such as interleukins IL-1, IL-2, IL-3, IL-6, IL-8 and tumour necrosis factor alpha (TNF) were found to play a major role in bone remodelling; moreover, interleukin-1 (IL-1) stimulates osteoclast function through its receptor on osteoclasts. It was also found that mechanical stresses due to orthodontic treatment increased the production of prostaglandin PGE and IL-1 beta in the periodontal ligament. Experiments were done on cats where one canine was tipped distally by $80 \mathrm{~g}$ of force from hours to days after which immunohisto chemistry and micro photometry experiments were done to measure the intensity of PGE and IL-1 beta which was found to be highest on the tension [5]. Other cytokines which are also involved in the acceleration of tooth movement are RANKL, which is a membrane-bound protein on the osteoclasts that bind 
to the RANK on the osteoclasts and causes osteoclastogenesis [6-8]. On the other hand, osteoprotegerin (OPG) competes with RANKL in binding to osteoclasts to inhibit osteoclastogenesis. The process of bone remodelling is a balance between (RANKL-RANK) system and OPG compound $[9,10]$. In relation to this, using biological molecules in the acceleration of tooth movement has been shown in two unique experiments in which it was demonstrated that the transfer of RANKL gene to the periodontal tissue induced prolonged gene expression for the enhancement of osteoclastogenesis and acceleration of tooth movements in rats. On the other hand, the transfer of OPG gene inhibited orthodontic tooth movements. In another study, it was found that teeth move faster in young patients than they do in adults. This can be attributed to the lower amount of RANKL/ OPG ratio in the gingival reticular fluid (GCF) in adult patients which is measured by the enzyme-linked immunosorbent assay method. RANK, OPG and root resorption showed a correlation during orthodontic teeth movement. It was observed that patients with root resorption seemed to produce a larger amount of RANKL in the compressed site.

Prostaglandin effect on tooth movement: Prostaglandins (PGs) are inflammatory mediators and can be considered paracrine hormones that impact nearby cells; thereby stimulating bone resorption by increasing the number of osteoclasts. In vivo and in vitro experiments were conducted to show clearly the relation between PGs, applied forces, and the acceleration of tooth movement. Yamasaki $[11,12]$ was among the first to investigate the effect of local administration of prostaglandin in rats and monkeys. In addition, experiments done in rats have shown that injections of exogenous PGE2 over an extended period of time caused acceleration of tooth movements. Furthermore, the acceleration rate was not affected by single or multiple injections or between different concentrations of the injected PGE2. However, root resorption was very clearly related to the different concentrations and number of injections given. It has also been shown that the administration of PGE2 in the presence of calcium stabilizes root resorption while accelerating tooth movement. Chemically produced PGE2 has also been studied in human trials with split-mouth experiments in the first premolar extraction cases. In these experiments, the rate of distal retraction of canines was 1.6-fold faster than the control side.

Effect of vitamin D3 on tooth movement: Vitamin D3 has also attracted the attention of scientists to its role in the acceleration of tooth movement; 1, 25 dihydroxycholecalciferol is a hormonal form of vitamin D and plays an important role in calcium homeostasis with calcitonin and parathyroid hormone (PTH). Another set of investigators [13] conducted an experiment where they have injected vitamin D metabolite on the PDL of cats for several weeks; it was found that vitamin D had accelerated tooth movement at $60 \%$ more than the control group due to the increase of osteoclasts on the pressure site as detected histological. A comparison between local injection of vitamin D and PGEs on two different groups of rats was also done. It was found that there is no significant difference in acceleration between the two groups. However, the number of osteoclasts on the pressure side which was injected by vitamin $\mathrm{D}$ was greater than on the PGE2 side. This indicates that vitamin D may be more effective in bone turnover.

PTH effect on tooth movement: PTH has shown to accelerate orthodontic tooth movement in rats, which was studied by continuous infusion of PTH (1 to $10 \mu \mathrm{g} / 100 \mathrm{~g}$ of body weight/day) implantation in the dorsocervical region which made the molars move 2 to 3 fold faster mesially by orthodontic coil spring [14] Some studies have shown that locally injected PTH induces local bone resorption and hence it is more advantageous to give PTH locally rather than systemically. It was also confirmed that a slowrelease local application of PTH was very efficient when a daily injection of PTH was dissolved in gel medium caused 1.6-fold faster acceleration of teeth compared to daily injection of PTH dissolved in saline which did not cause any acceleration.

\section{Surgical approach}

The surgical technique to enhance the rate of tooth movement has been documented in many case reports previously. It is a clinically effective technique used for adult patients, where duration of orthodontic treatment may be critical in selective groups of patients. The PDL and alveolar bone remodelling are important parameters in tooth movement, and bone turnover is known to increase after bone grafting, fracture, and osteotomy. Various surgical approaches that have been tried to accelerate tooth movement include osteotomy, corticotomy and piezocision technique.

Corticotomy and osteotomy: A corticotomy is defined as a surgical procedure where only the outer cortical bone is cut, perforated or modified. The medullar bone is left intact. This is in contrast to an osteotomy where the surgical cut perforates both cortical and medullar bone. It was first tried in orthodontics by Kole [15], where tooth movements were achieved between 6 and 12 months. The technique was further used by others, like Grenerson et al. [16] who used this for open bites treatments. In 2001, Wilcko et al. [17] reported that the acceleration of tooth movement is not due to the bony block movement as postulated by Kole [15]; but rather a process of bone remodelling at the surgical site, which was called regional acceleratory phenomenon (RAP). He developed and patented techniques which were called accelerated oestrogenic orthodontics (AOO) and periodontal accelerated oestrogenic orthodontics. RAP was modified by adding bio-absorbable grafting material over the injured bone to enhance healing. This technique is reported to have postoperative stability and improved retention; however there is a constant need for more research. The only pitfall of these surgical techniques is their invasive nature and the fact that acceleration was seen only in the first 3 to 4 months after which it declines with time.

\section{Advantages}

a. It has been proven successfully by many authors to accelerate tooth movement.

b. Bone can be augmented, thereby preventing periodontal defects, which might arise, as a result of thin alveolar bone. 


\section{Disadvantages}

a. High morbidity associated with the procedure.

b. Invasive procedure.

c. Chances of damage to adjacent vital structures.

d. Post-operative pain, swelling, chances of infection, vascular necrosis.

e. Low acceptance by the patient.

Piezocision technique: One of the latest techniques in accelerating tooth movement is the Piezocision technique. Dibart [18] was among the first to apply the piezocision technique which starts with primary incision placed on the buccal gingival followed by incisions by Piezosurgical knife to the buccal cortex. Piezocision technique did not cause any periodontal damage as reported by Hassan [19]. Another benefit of this technique is that it can be used with Invisalign, which gives a better aesthetic appearance and less treatment time as reported by Keser. Piezocision is a promising tooth acceleration technique because of its various advantages in periodontal, aesthetic, and orthodontic aspects.

\section{Advantages}

a. Minimally invasive.

b. Better patient acceptance.

\section{Disadvantages}

a. Risk of root damage, as incisions and corticotomy if "blindly" done.

To reduce the risk of root damage, however, Jorge et al in 2013, 2014 suggested a method, called MIRO (Minimally Invasive Rapid Orthodontic procedure) by using a metal wire as a guide to placement of the incisions for corticotomy cuts. He placed metal guides in between each tooth, perpendicular to the main arch wire, and took digital radiographs, to ensure that the metal guides did not project over the tooth roots. Once this was confirmed, incisions and piezoelectric corticotomy were done using pins as a guide.

\section{Physical approach}

Surgical methods, regardless of technique, are still invasive to some degree and hence have their associated complications. Hence, non-invasive methods have come into light. The concept of using physical approaches came from the idea that applying orthodontic forces causes bone bending (bone bending theory) and bioelectrical potential develops. The concave site will be negatively charged attracting osteoclasts and the convex site will be positively charged attracting osteoclasts as detected by Zen go in his measurements on dog alveolar bone. The bioelectrical potential is created when there is application of discontinuous forces, which leads to the idea of trying cyclic forces and vibrations. It has been found that applying vibrations for different duration per day accelerated tooth movements between $15 \%$ and $30 \%$ in animal experiments. These modalities include lasers, vibration, direct electric current etc.
Low-level laser therapy: Photo-bio modulation or low-level laser therapy (LLLT) is one of the most promising approaches today. Laser has a bio-stimulatory effect on bone regeneration, which has been shown in the midpalatal suture during rapid palatal expansion [20] and also stimulates bone regeneration after bone fractures the extraction site [21,22] Laser light has been seen to stimulate the proliferation of osteoclast and fibroblasts, and thereby affects bone remodelling and accelerates tooth movement. The mechanism involved in the acceleration of tooth movement is by the production of ATP and activation of cytochrome $\mathrm{C}$, which was seen when lowenergy laser irradiation enhanced the velocity of tooth movement via RANK/RANKL and the macrophage colony-stimulating factor and its receptor expression.

In 2004, it was Cruz et al. who carried out a human study on the effect of low-intensity laser therapy on orthodontic tooth movement. They showed that the irradiated canines were retracted at a rate $34 \%$ greater than the control canines over 60 days.

Animal experiments have shown that low-level laser can accelerate tooth movement. Furthermore, clinical trial attempts were made in which different intensities of laser were used and different results were obtained. Low-level laser therapy can be a very useful technique for acceleration of tooth movement since it increases bone remodelling without causing any side effect to the periodontium. Laser wavelength of $800 \mathrm{~nm}$ and output power of $0.25 \mathrm{mw}$ has indicated significant stimulation of bone metabolism, rapid ossification and also acceleration of tooth movement to 1.5 fold in rat experiments. Lately in a clinical trial study, the laser wavelength they have used in a continuous wave mode at $800 \mathrm{~nm}$, with an output of $0.25 \mathrm{mw}$ and exposure of $10 \mathrm{~s}$ was found to accelerate tooth movement at 1.3 fold higher than the control. In another study done by Kau on 90 subjects ( 73 test subjects and 17 controls), there was $1.12 \mathrm{~mm}$ change per week in the test subjects versus $0.49 \mathrm{~mm}$ in the control group. Having said this, there are a lot of contradictory results related to the LLLT. Therefore, more experiments are needed to differentiate the optimum energy, wavelength, and the optimum duration for usage.

Direct electric current effect on tooth movement: Direct electric current application is a promising approach to accelerate tooth movement. This technique was tested only on animals by applying direct current to the anode at the pressure sites and cathode at the tension sites (by 7V) thus generating local responses and acceleration of bone remodelling as shown by group of investigators [23]. Their studies were more successful than the previous attempts because electrodes were placed as close as possible to the moving tooth. The bulkiness of the devices and the source of electricity made it difficult to be tested clinically. Several attempts were made to develop bio-catalytic fuel cells to generate electricity intraoral by the use of enzymes and glucose as fuel. Further development of the direct electric device and the biocatalytic fuel cells is needed in order to test this clinically. 


\section{Clinical Application for the Future}

The administration of exogenous biological molecules to accelerate tooth movement during orthodontic treatments has been intensively tested on animal experiments. However, clinical trials on humans are limited since they are to be administered local injections which can be painful and discomforting to the patients. Also, their side effects were not tested for long periods of time. However, administrations of certain molecules have shown promising results; for example, cytokine, PTH, vitamin D, and RANKL/RANK/OPG system play an important role in bone remodelling and tooth movement.

In the physical approach, the low level laser therapy is the most promising method; however, contradictory results are seen. This is due to the different energies, duration and experimental design. Furthermore, most of these experiments were done in only few weeks, which is a very short time to notice any side effects. The surgical approach is the most clinically used and most tested with known predictions and stable results. However, it is invasive, aggressive and costly and patients are not open to the idea of surgery unless it is the only option needed to have a good occlusion. Piezocision technique is one of the newest techniques in accelerating tooth movement which has good clinical outcome and is considered the least invasive in the surgical approach.

\section{Conclusion}

For the longest time, patients have been asking for quick, effective and convenient orthodontic treatment. After years of research and innovation, we can conclude that we now have methods and resources of superior quality which not only enable us to provide quick, but also comfortable orthodontic treatment to both children and adults. The current methods such as piezocision, lasers and vibration have eliminated the invasive nature of previous procedures used to achieve the Regional Acceleratory Phenomenon. Such methods come with additional advantages such as reduced rates of relapse, less orthodontic pain as well as root resorption. Although these techniques have drawbacks and uncertainties associated with them, they are a step closer to quicker orthodontic treatment making them the next frontier for orthodontics and its success.

\section{References}

1. Wilcko WM, Wilcko T (2008) An evidence based analysis of periodontally accelerated orthodontic and osteogenic techniques: A Synthesis of Scientific Perspectives. Seminars in Orthodontics 14(4): 305-316.

2. Shailesh S, Krishna Nayak US, Vivek B, Nayak A (2014) Acclerated Orthodontics-A Review. Int J Scient Study 1(5): 35-39.

3. Leiker BJ, Nanda RS, Currier GF, Howes RI (1995) The effects of exogenous prostaglandins on orthodontic tooth movement in rats. Am J Orthod Dentofacial Orthop 108(4): 380-388.

4. Krishnan V, Davidovitch Z (2006) The effect of drugs on orthodontic tooth movement. Orthod Craniofac Res 9(4): 163-171.

5. Saito M, Saito S, Ngan PW, Shanfeld J (1991) Interleukin 1 beta and prostaglandin $\mathrm{E}$ are involved in the response of periodontal cells to mechanical stress in vivo and in vitro. Am J Orthod Dentofacial Orthop 99(3): 226-240.

6. Udagawa N, Takahashi N, Jimi E, Matsuzaki K (1999) Osteoblasts/stromal cells stimulate osteoclast activation through expression of osteoclast differentiation factor/RANKL but not macrophage colony-stimulating factor: receptor activator of NF-kappa B ligand. Bone 25(5): 517-523.

7. Drugarin DDM, Negru S, Cioace R (2003) RANKL/RANK/OPG molecular complex-control factors in bone remodeling. TMJ 53: 296-302.

8. Kim SJ, Kang YG, Park JH, Kim EC, Park YG, et al. (2013) Effects of lowintensity laser therapy on periodontal tissue remodeling during relapse and retention of orthodontic ally moved teeth. Lasers Med Sci 28(1): 325-333.

9. Simonet WS, Lacey DL, Dunstan CR, Kelley M, Chang MS, et al. (1997) Osteoprotegerin: a novel secreted protein involved in the regulation of bone density. Cell 89(2): 309-319.

10. Oshiro T, Shiotani A, Shibasaki Y, Sasaki T (2002) Osteoclast induction in periodontal tissue during experimental movement of incisors in osteoprotegerin-deficient mice. Anat Rec 266(4): 218-225.

11. Yamasaki K, Miura F, Suda T (1980) Prostaglandin as a mediator of bone resorption induced by experimental tooth movement in rats. J Dent Res 59(10): 1635-1642.

12. Yamasaki K, Shibata Y, Fukuhara T (1982) The effect of prostaglandins on experimental tooth movement in monkeys (Macacafuscata). J Dent Res 61(12): 1444-1446.

13. Collins MK, Sinclair PM (1988) The local use of vitamin D to increase the rate of orthodontic tooth movement. Am J Orthod Dentofacial Orthop 94(4): 278-284

14. Soma S, Iwamoto M, Higuchi Y, Kurisu K (1999) Effects of continuous infusion of PTH on experimental tooth movement in rats. J Bone Miner Res 14(4): 546-554

15. Kole H (1959) Surgical operations on the alveolar ridge to correct occlusal abnormalities. Oral Surg Oral Med Oral Pathol 12(5): 515-529.

16. Generson RM, Porter JM, Zell A, Stratigos GT (1978) Combined surgical and orthodontic management of anterior open bite using corticotomy. J Oral Surg 36(3): 216-219.

17. Wilcko WM, Wilcko T, Bouquot JE, Ferguson DJ (2001) Rapid orthodontics with alveolar reshaping: two case reports of decrowding. Int J Periodontics Restorative Dent 21(1): 9-19.

18. Dibart S, Surmenian J, Sebaoun JD, Montesani L (2010) Rapid treatment of Class II malocclusion with piezocision: two case reports. Int J Periodontics Restorative Dent 30(5): 487-493.

19. Mittal SKS, Singla A (2011) Piezocision assisted orthodontics: a new approach to accelerated orthodontic tooth movement. Innovative Dentistry 1: 1 .

20. Saito S, Shimizu N (1997) Stimulatory effects of low-power laser irradiation on bone regeneration in midpalatal suture during expansion in the rat. Am J Orthod Dentofacial Orthop 111(5): 525-532.

21. Trelles MA, Mayayo E (1987) Bone fracture consolidates faster with lowpower laser. Lasers Surg Med 7(1): 36-45.

22. Takeda Y (1988) Irradiation effect of low-energy laser on alveolar bone after tooth extraction. Experimental study in rats. Int J Oral Maxillofac Surg 17(6): 388-391.

23. Davidovitch Z, Finkelson MD, Steigman S, Shanfeld JL (1980) Electric currents, bone remodeling, and orthodontic tooth movement. II. Increase in rate of tooth movement and periodontal cyclic nucleotide levels by combined force and electric current. Am J Orthod 77(1): 33-47. 